\title{
Electroimpedance Spectroscopy for the Measurement of the Dielectric Properties of Sodium Chloride Solutions at Different Glucose Concentrations
}

\author{
Stefano Sbrignadello, Andrea Tura, and Paolo Ravazzani \\ Institute of Biomedical Engineering, National Research Council, Corso Stati Uniti 4, 35127 Padova, Italy \\ Correspondence should be addressed to Stefano Sbrignadello; stefano.sbrignadello@isib.cnr.it
}

Received 20 April 2012; Revised 20 July 2012; Accepted 24 July 2012

Academic Editor: Zhifeng Shao

Copyright (C) 2013 Stefano Sbrignadello et al. This is an open access article distributed under the Creative Commons Attribution License, which permits unrestricted use, distribution, and reproduction in any medium, provided the original work is properly cited.

We investigated possible variations of impedance values in samples of sodium chloride solution (sodium chloride $0.9 \%$ ) with glucose at different concentrations, ranging from 5000 to around $75 \mathrm{mg} / \mathrm{dL}$. The sodium chloride solution (either saline physiological solution) was chosen since it has similarities to blood but no cell components, which may be confounding factors in this study. Special focus was on the effect of stirring and of temperature variations on the impedance spectrum of samples at different glucose concentrations. We found that variations in glucose concentration directly affect the impedance modulus of the sample both in static conditions and in dynamic conditions due to stirring, as well as at both room temperature and at increased temperature. In fact, even if the impedance variations were often small (around 3-4 $\mathrm{m} \Omega$ per $\mathrm{mg} / \mathrm{dL}$ ), they were usually clearly measurable. These findings may be the basis for possible development of a new approach, based on impedance technology, for the noninvasive monitoring of glycaemia.

\section{Introduction}

The measurement of dielectric properties of biological tissues through electroimpedance spectroscopy techniques has been performed in several medical and clinical applications [1, 2]. In recent years, electroimpedance spectroscopy has been suggested as a noninvasive approach to determine glycaemia, as reported in several reviews [3-6].

In [7] the authors showed that variations in blood glucose concentration determine significant changes in the impedance of a subject's skin and underlying tissues due to biochemical reactions across the membrane of erythrocytes triggered by variations in glucose concentration. In other studies, however, impedance variations were found in glucose-water solutions with different glucose concentrations, although no cell component was present [8]. Similar results were found in our previous study [9], where we investigated the impedance variations in a few glucose-water, glucose-sodium chloride, and glucose-blood samples.

The main aim of this study was to more deeply analyze the changes in the impedance of glucose-sodium chloride solutions at $0.9 \%$ as a function of glucose concentration in a range upto $5000 \mathrm{mg} / \mathrm{dL}$; the influence of change in the solution temperature and the effects due to the stirring of the sample (dynamic conditions) have been also investigated. The saline solution was chosen as it has an osmotic pressure close to that of plasma and conductivity similar to that of blood. This approach could represent a step forward in the understanding of the role of glucose in the dielectric property changes of biological fluids. The lack of any cellular component would allow us to clarify the direct effect of glucose, excluding any cellular process. These results could be of some help in better understanding the realistic possibility to use approaches for glycaemia monitoring based on the measurement of glucoseinduced dielectric property variations. 


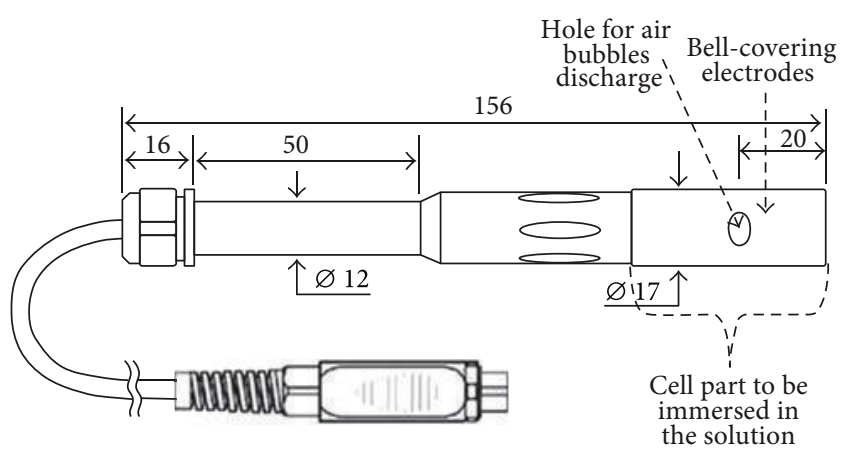

Figure 1: Scheme of the Delta OHM Probe Sp06T.

\section{Experimental Section}

2.1. Preparation of Samples. For the preparation of the samples, sodium chloride $0.9 \%$ (Baxter) and D-glucose $(99.5 \%$, Fluka) was used. First, $500 \mathrm{~mL}$ of sodium chloride $0.9 \%$ (saline physiological solution) was prepared in a glass cylinder, with the addition of $25 \mathrm{~g}$ of glucose to reach the final concentration of $5000 \mathrm{mg} / \mathrm{dL}$. The sample was afterwards diluted, by eliminating $25 \mathrm{~mL}$ of the sample and adding the same quantity of pure saline physiological solution. The process was iterated several times, and, at the end, seven samples were prepared, with glucose concentration values of $\sim 78, \sim 156, \sim 312,625,1250,2500$, and $5000 \mathrm{mg} / \mathrm{dL}$. A sample of pure saline physiological solution was also prepared as blank control.

\subsection{Impedance Equipment and Measurement Approach.} Impedance measures were performed by means of a Solartron 1260 impedance analyzer (Solartron Analytical). For the measurement cell a probe from Delta OHM, SP06T model, was chosen (Figure 1). The cell $k$-factor was $K=0.7$ and the measurement range was from $5 \mu \mathrm{S} / \mathrm{cm}$ to $200 \mathrm{mS} / \mathrm{cm}$.

The impedance of the samples was measured in the $10^{5}-10^{7} \mathrm{~Hz}$ frequency range, in five frequency points for each decade. In this study, lower frequency values were not considered to avoid having possible confounding factors related to electrode polarization. The cell was characterized by four platinum electrodes (Figure 2) for possible separation between stimulation and sensing terminals and consequent minimization of possible secondary effects (inductance of cables or parasitic capacitances) [10].

However, in this study, we did not perform measures above $10 \mathrm{MHz}$ (i.e., not very high frequencies), thus the weight of the possible secondary effects was supposed to be negligible. On the other hand, when the sample to be measured has quite high conductivity, for better accuracy and precision it may be convenient to use electrodes at sufficiently high distance. For this reason we decided to use the external couple of electrodes not only for stimulation but also for sensing. The tests were performed by applying a $100 \mathrm{mV}$ r.m.s. voltage to the sample through the external couple of electrodes.

2.3. Experiments. On each of the samples at the different glucose concentrations, we performed the following

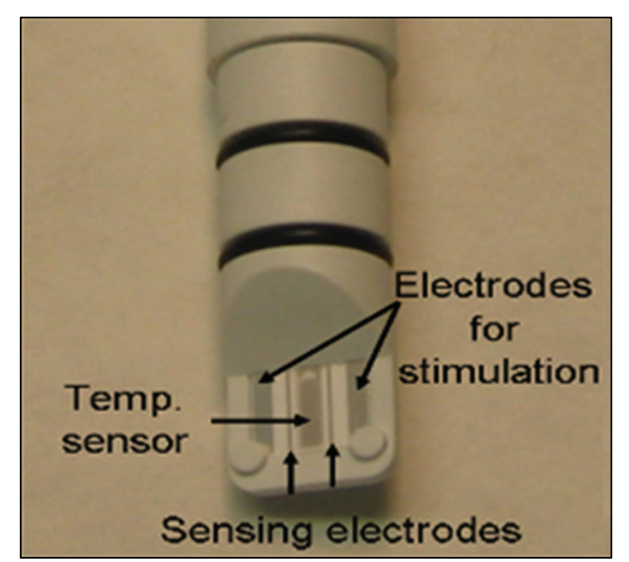

FIGURE 2: Details of the four plain platinum electrodes of the Delta OHM Probe Sp06T.

experiments: (i) the impedance measure was performed on the sample at room temperature (around $22^{\circ} \mathrm{C}$ ), in static condition; (ii) to obtain dynamic conditions, the sample was stirred through an electromagnetic microstirrer (Velp Scientifica), while the impedance measure was carried on; (iii) Then, the impedance measures, both in static and dynamic conditions, as reported in (i) and (ii), were performed after heating the sample, thus reaching different stable temperatures, selected in a wide range including also the typical temperatures of human body fluids $(32,37,42$, and $47^{\circ} \mathrm{C}$ ); heating was obtained by means of a heating plate (Velp Scientifica) and checked through a thermometer with $\pm 0.3^{\circ} \mathrm{C}$ accuracy (Checktemp ${ }^{\circ}$, Hanna Instruments).

Each experiment, for each sample, in both static and dynamic conditions, and at the different studied temperature, was performed four times: after each experiment, the measurement cell was cleaned before immersing it again into the same sample. Each impedance value presented in the results section is the average between the four measures on the same sample (in each specific condition), unless otherwise specified.

2.4. Statistical Analysis. On each couple of measurement sets (static and dynamic sets), at the different temperatures, we performed a nonparametric test, that is, the Wilcoxon Signed Rank test. In fact, we aimed to assess possible differences, on average, between the values in static and dynamic conditions, for each temperature (studied values: impedance modulus at $1 \mathrm{MHz}) . P<0.05$ was considered for statistically significant difference.

\section{Results}

The impedance modulus of the samples at room temperature is reported in Figure 3(a). The modulus increases for the increasing glucose concentration values in all the studied frequency range, even though the differences for low glucose concentrations were modest. Similar results were found for the set of experiments performed at the different 


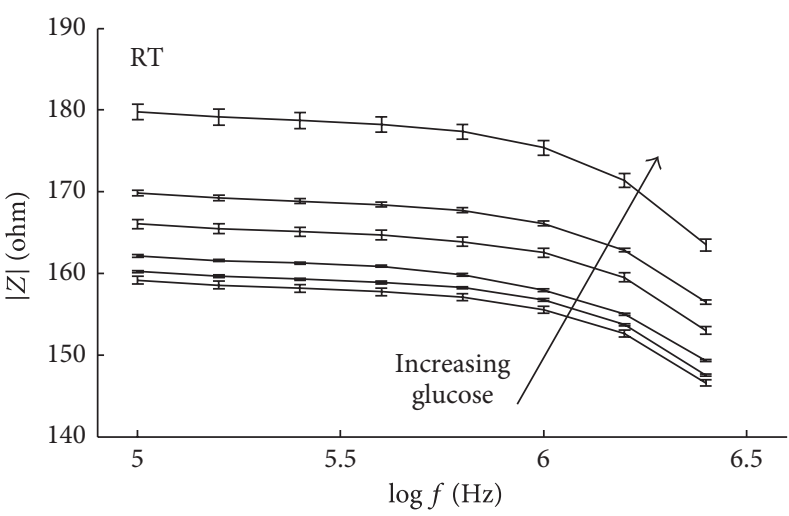

(a)

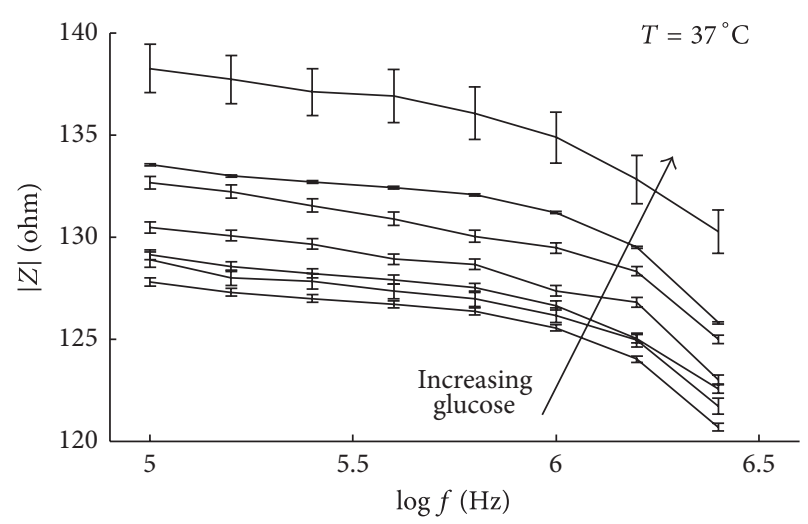

(b)

FIGURE 3: Impedance modulus of samples of physiological solution alone and with added glucose at concentrations from 5000 to nearly $78 \mathrm{mg} / \mathrm{dL}$, at room temperature (a) and at $37^{\circ} \mathrm{C}(\mathrm{b})$. Data reported are mean $\pm \mathrm{SE}$.

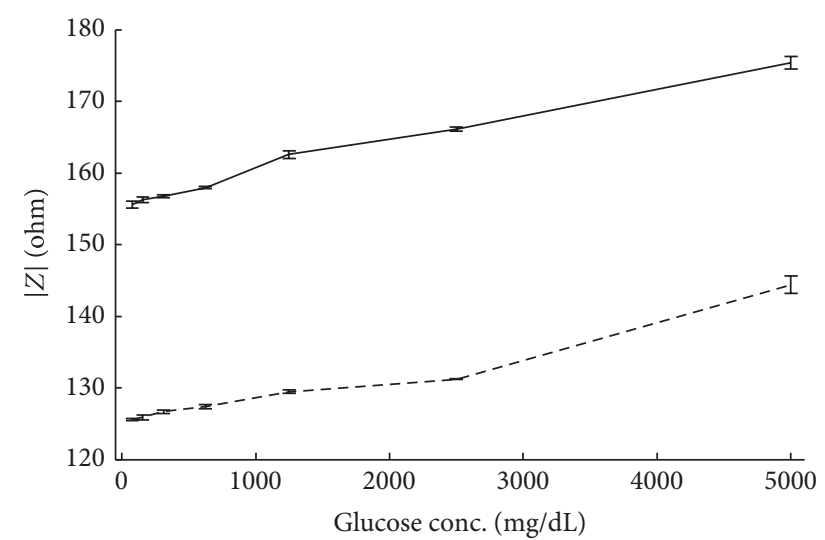

FIGURE 4: Impedance modulus of the samples as a function of glucose concentration at $1 \mathrm{MHz}$. Results at the two different temperatures are shown: solid curve: room temperature; dashed curve: $37^{\circ} \mathrm{C}$. Data reported are mean $\pm \mathrm{SE}$.

temperatures: for instance, Figure 3(b) shows the impedance modulus over all the studied frequency range at $37^{\circ} \mathrm{C}$.

Figure 4 shows the impedance values measured at $1 \mathrm{MHz}$ (value in the middle of the studied frequency range) as a function of the glucose concentration at room temperature and at $37^{\circ} \mathrm{C}$. The two curves show practically a parallel trend, with a decrease of the impedance values in the samples at higher temperature. Such decrease remains essentially constant at any glucose concentration value. The slopes of the curves, as estimated by linear regression, are of about 4.6 and $3.2 \mathrm{~m} \Omega /(\mathrm{mg} / \mathrm{dL})$ for the samples at room temperature and $37^{\circ} \mathrm{C}$, respectively. The $R$ values of the linear regression were found of 0.98 and 0.99 , respectively.

It should be acknowledged that the samples at lower glucose concentrations, typical of the physiological glycemic levels, were not clearly distinguishable (RT: $78 \mathrm{mg} / \mathrm{dL}: 125.54$ $\pm 0.16 \Omega$ (mean $\pm \mathrm{SE}) ; 156 \mathrm{mg} / \mathrm{dL}: 125.86 \pm 0.36 \Omega$ ); however, when comparing the $78 \mathrm{mg} / \mathrm{dL}$ sample with the

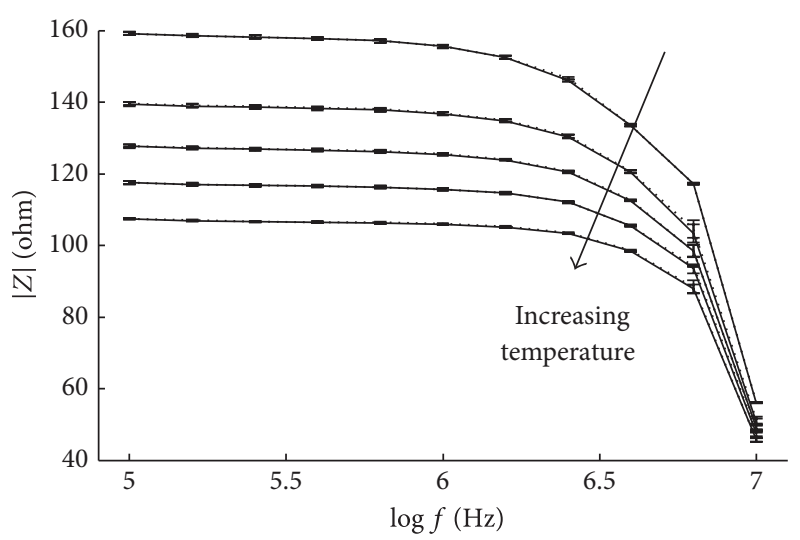

FIGURE 5: Impedance modulus spectrum for the samples at $78 \mathrm{mg} / \mathrm{dL}$, in static (dotted curve) and dynamic (solid curve) conditions at room temperature and at $32,37,42$, and $47^{\circ} \mathrm{C}$ (from top to bottom). Data reported are mean \pm SE.

$312 \mathrm{mg} / \mathrm{dL}$ sample (concentration values still in the pathophysiological range), the difference was clearly significant $(312 \mathrm{mg} / \mathrm{dL}: 126.64 \pm 0.21 \Omega, P \leq 0.02$ with the Wilcoxon Signed Rank test). Similar considerations hold for the samples at $37^{\circ} \mathrm{C}$. It should also be noted that in this study the experimental setup was not optimized for the measurements at low glucose concentration values, thus it cannot be excluded that performance at such values could be improved.

Figure 5 shows the results obtained under static and dynamic conditions for the sample at $78 \mathrm{mg} / \mathrm{dL}$ at all the temperatures tested. For each temperature, the impedance in static and dynamic condition is virtually the same, as mirrored by the static and dynamic related curves virtually overlapped. Similar results were obtained for the other samples, at different glucose concentrations (not shown).

Indeed, this is confirmed by the statistical test, which found no statistically significant difference between the impedance modulus (at $1 \mathrm{MHz}$ ) in static and dynamic 
TABle 1: Values at $1 \mathrm{MHz}$ for the $78 \mathrm{mg} / \mathrm{dL}$ glucose sample at different temperatures in static and dynamic conditions. $P$ range for the different static/dynamic couple of values: $0.14-0.72$ (i.e., not significant).

\begin{tabular}{lcccccccccc}
\hline $\begin{array}{l}\text { Temperature } \\
\text { Status }\end{array}$ & Static & Dynamic & Static & Dynamic & Static & Dynamic & Static & Dynamic & Static & Dynamic \\
\hline Test 1 & 156,11 & 156,10 & 136,38 & 136,08 & 125,34 & 125,27 & 115,99 & 116,25 & 105,99 & 105,81 \\
Test 2 & 155,71 & 155,69 & 137,38 & 136,94 & 125,47 & 125,78 & 115,73 & 115,56 & 106,23 & 105,62 \\
Test 3 & 155,35 & 155,42 & 137,07 & 137,12 & 125,67 & 124,96 & 115,48 & 115,48 & 106,03 & 105,73 \\
Test 4 & 155,08 & 155,11 & 136,91 & 136,84 & 125,67 & 125,67 & 115,42 & 115,39 & 105,60 & 105,90 \\
\hline
\end{tabular}

conditions, for all the samples at the different glucose concentrations, at each of the different temperatures. Table 1 reports the impedance modulus of the sample at $78 \mathrm{mg} / \mathrm{dL}$ glucose, at the different temperatures.

\section{Discussion}

The noninvasive measurement of glycaemia is a topic of great interest and is rapidly changing; novelties have emerged frequently in the last years. Nevertheless, in spite of some encouraging results and the efforts made over the past years, there is no device available at present for use in clinical practice. A possible explanation might be the combination between the specific features of each method and the specific characteristics of diabetic patients, which make them respond differently to physical and chemical methods when compared to their healthy counterparts. The most recent device prototypes appear promising, but because of the failure of previous projects, the assessment of such new devices must be done with extreme caution $[3,11,12]$.

One of the promising approaches for noninvasive measurement of glycaemia is electroimpedance spectroscopy. Some device prototypes have been developed based on this approach, and one of them also received the CE approval, but its diffusion in the market was prevented by some concerns about its actual performances [13]. A new company seems to be working on a similar device [14], but at the moment no device is available.

In [7] authors claimed that the measurement of glycaemia through electroimpedance spectroscopy is possible as variations in blood glucose concentration induce some transportation phenomena of electrolytes through the cell membrane, and as a consequence of this process, variations in the dielectric properties of the medium can be observed. The most relevant phenomenon seems to be the plasma sodium concentration lowering in the presence of hyperglycaemia [15]. In [7] it was claimed that these effects are entirely responsible for the impedance variations of blood and underlying tissues, since glucose variations do not directly affect the dielectric properties of the investigated medium (at least in the $\mathrm{MHz}$ band). However, some recent studies contradict these findings: in $[8,9]$ the dielectric properties of glucose-water solutions were found different for glucose concentration values varying within the physiological range. In particular, the impedance modulus increased for increasing glucose concentrations within the $1 \mathrm{kHz}-1 \mathrm{MHz}$ band. However, in our previous study [9], for each type of solution only a few samples were studied, and only in static conditions, at room temperature.

In this study, impedance changes of sodium chloride solutions at different glucose concentration values were analyzed more deeply. The interest for the sodium chloride $0.9 \%$ solution is due to the fact that it has an osmotic pressure extremely close to that of plasma, and it also has conductivity similar to that of blood: in fact, we also measured sodium chloride $0.9 \%$ conductivity with a simple conductivity meter (Orion) and found $15.60 \mathrm{mS} / \mathrm{cm}$ (at room temperature), which is not far from values reported for blood in the literature [16].

Blood is a very complex tissue, liable to sudden time changes in the several elements (proteins, hormones, etc.) and ion concentration due to many different factors [17, 18]. From our previous studies and from what emerges in the literature, it is clear that these characteristics make it extremely difficult to isolate the behavior of the various components that contribute to the various measures applicable to noninvasive monitoring of blood glucose [11]. For this reasons in this study we have tried to isolate two fundamental physical-chemical characteristics in the impedance spectroscopy analysis: the conductivity and the osmotic pressure, using for this proposal solutions comparable with blood only in these two particular features.

Solutions at different glucose concentrations were examined at five different temperatures, also including the temperature typical of human body fluids $(22,32,37,42$, and $47^{\circ} \mathrm{C}$ ), both in static and dynamic conditions, the latter being more similar to what occurs in an in vivo context. The results showed that the impedance modulus of the solution samples was affected by the glucose concentration at all the studied frequencies, confirming that variations in glucose concentration even in the physiological range influence the dielectric properties of solutions. However, the changes in dielectric properties were small at any tested concentrations. This is particularly true at glucose concentrations in the physiopathological range (from about 100 to $300-400 \mathrm{mg} / \mathrm{dL}$ ), where changes of a few $\mathrm{m} \Omega /(\mathrm{mg} / \mathrm{dL})$ were found at room temperature. These results were also confirmed when the temperature of the sample was increased. In these cases, as expected, the values of impedance as a function of the glucose concentration were lower than those at $22^{\circ} \mathrm{C}$ (for the experiments at $37^{\circ} \mathrm{C}$, average difference of about $35.9 \Omega$ ), but a similar trend was observed (see Figure 4).

As regards the possible differences between static and dynamic conditions, some studies can be found in the scientific literature about possible differences in the impedance 
spectrum between these two conditions, but they are typically focused on the investigation of the main characteristics of ion-exchange membranes $[19,20]$. To our knowledge, there is only one study [21] in the literature that deals with the problem of glucose measurement using an impedancebased approach, with the investigation of both static and dynamic conditions. In the study [21] the impedance of some animal blood samples at different glucose concentrations was analysed, both in static and dynamic conditions, at the same temperature. At some glucose concentrations, the sample in dynamic conditions showed a slight decrease in the impedance value compared to the sample in static conditions; however, at other glucose concentration values, an opposite behaviour was found. The authors were unable to provide a clear explanation of these findings. In our study, as can be observed in Figure 5 and Table 1, there was no difference between static and dynamic conditions in each sample at each temperature tested. In fact, the solutions used for our experiments include an ionic solute $(\mathrm{NaCl})$ completely solvated by polar solvent molecules $\left(\mathrm{H}_{2} \mathrm{O}\right)$, and also the complete dissolution of glucose contributes to the homogeneity of the solution. Our hypothesis is that in experimental conditions involving relatively small volumes $(<100 \mathrm{~mL})$ of highly homogeneous solutions, the agitation of the sample tends not to influence the ions mobility, and hence the flow of the electric current. Besides, the lack of differences (or the presence of negligible differences) in dielectric measurements between static and dynamic conditions was also reported in our previous study [22], though in that case the experimental setup was completely different and only simple conductivity measurements were performed, instead of impedance spectra measurements. In any case, we acknowledge that the discrepancies between our studies and the study [21] in the findings related to the possible differences between static and dynamic data may be due to the differences in the type of solutions analyzed in each study.

As regards the experimental setup of this study, it must be noted that the measurement probe that we selected is generally used in simple conductivity analysis, and hence it may be not completely adequate for impedance measurements. We selected that probe for the presence of platinum electrodes, despite the relatively low cost. However, the most interesting results (i.e., those reported in Results) were obtained in a frequency range where the phase of the measured impedance was small, or even almost zero (data not shown). Clearly, when the phase was zero the impedance reduced to conductance, and hence the probe was certainly used properly at those frequency values.

The results presented here might have been affected by some electrode polarization phenomena. However, it is well known that the electrode polarization is more relevant at low frequency values. Indeed, it is unlikely at frequencies above a hundred of $\mathrm{kHz}$. Moreover, electrode polarization has effects more pronounced on the capacitance rather than on the conductance of the investigated medium, as explained in [23] and in our study, the major findings were observed at frequencies where the capacitance was small or negligible (phase of the impedance almost zero in the studied frequency range). Finally, we used platinum electrodes, which are less prone to electrode polarization phenomena compared to other materials [24-26].

In conclusion, we found that variations of glucose concentration in sodium chloride solution samples directly affect the impedance of the samples, even in the absence of other mechanisms that may occur in the presence of cells and tissues interactions, which may strengthen the differences, but may also act as confounding factors. The mechanisms responsible for the resistive effect of glucose in ionic solutions are not known. Many problems related to carbohydrates are still far from properly understood, such as the behavior of glucopyranose (circular glucose) in aqueous solution. The glucose-water solution has been studied by both force-field molecular dynamics methods and quantum-chemical solvation, and most recently with the help of multisite models [27, 28]. It is known that the solubility of glucose is dependent on the intermolecular attractions between glucose-glucose and glucose-solvent molecules. Molecular dynamics simulations have shown that water acts as a solubility enhancer that increases the mobility and monodispersity of the system which leads to an increase in glucose solubility in ionic liquids with a consequent increase interaction between solute (glucose) and solvent (water) [29]. However, we do not know if the solvation shell of glucose or the dynamics of solutesolvent interactions may be responsible for the resistive effect observed empirically. Other studies will be needed in this area, perhaps by comparing the relationship between the solvation-solubility of other carbohydrates in ionic solutions, with the possible resulting resistive effect.

We can also claim that there are no impedance differences between static and dynamic conditions, at the different temperatures analyzed, and for the limited volumes and types of solutions used in the study. Though the impedance differences directly produced by glucose variations were small, these variations may be useful for future new approaches for noninvasive glycaemia monitoring.

\section{Acknowledgment}

The authors thank Dr. G. Pacini for his useful comments and advice.

\section{References}

[1] U. G. Kyle, I. Bosaeus, A. D. De Lorenzo et al., "Bioelectrical impedance analysis - part I: review of principles and methods," Clinical Nutrition, vol. 23, no. 5, pp. 1226-1243, 2004.

[2] S. Mattsson and B. J. Thomas, "Development of methods for body composition studies," Physics in Medicine and Biology, vol. 51, no. 13, pp. R203-R228, 2006.

[3] A. Tura, A. Maran, and G. Pacini, "Non-invasive glucose monitoring: assessment of technologies and devices according to quantitative criteria," Diabetes Research and Clinical Practice, vol. 77, no. 1, pp. 16-40, 2007.

[4] A. Sieg, R. H. Guy, and M. B. Delgado-Charro, "Noninvasive and minimally invasive methods for transdermal glucose monitoring," Diabetes Technology and Therapeutics, vol. 7, no. 1, pp. 174-197, 2005. 
[5] O. S. Khalil, "Non-invasive glucose measurement technologies: an update from 1999 to the dawn of the new millennium," Diabetes Technology and Therapeutics, vol. 6, no. 5, pp. 660-697, 2004.

[6] D. C. Klonoff, "Noninvasive blood glucose monitoring," Diabetes Care, vol. 20, no. 3, pp. 433-437, 1997.

[7] A. Caduff, E. Hirt, Y. Feldman, Z. Ali, and L. Heinemann, "First human experiments with a novel non-invasive, nonoptical continuous glucose monitoring system," Biosensors and Bioelectronics, vol. 19, no. 3, pp. 209-217, 2003.

[8] J.-H. Park, C.-S. Kim, B.-C. Choi, and K.-Y. Ham, "The correlation of the complex dielectric constant and blood glucose at low frequency," Biosensors and Bioelectronics, vol. 19, no. 4, pp. 321-324, 2003.

[9] A. Tura, S. Sbrignadello, S. Barison, S. Conti, and G. Pacini, "Impedance spectroscopy of solutions at physiological glucose concentrations," Biophysical Chemistry, vol. 129, no. 2-3, pp. 235-241, 2007.

[10] S. A. Awan and B. P. Kibble, "Towards accurate measurement of the frequency dependence of capacitance and resistance standards up to $10 \mathrm{MHz}$,' IEEE Transactions on Instrumentation and Measurement, vol. 54, no. 2, pp. 516-520, 2005.

[11] A. Tura, "Noninvasive glycaemia monitoring: Background, traditional findings, and novelties in the recent clinical trials," Current Opinion in Clinical Nutrition and Metabolic Care, vol. 11, no. 5, pp. 607-612, 2008.

[12] A. Ciudin, C. Hernández, and R. Simó, "Non-invasive methods of glucose measurement: current status and future perspectives," Current Diabetes Reviews, vol. 8, no. 1, pp. 48-54, 2012.

[13] I. M. E. Wentholt, J. B. L. Hoekstra, A. Zwart, and J. H. DeVries, "Pendra goes Dutch: lessons for the CE mark in Europe," Diabetologia, vol. 48, no. 6, pp. 1055-1058, 2005.

[14] A. Caduff, F. Dewarrat, M. Talary, G. Stalder, L. Heinemann, and Y. Feldman, "Non-invasive glucose monitoring in patients with diabetes: a novel system based on impedance spectroscopy," Biosensors and Bioelectronics, vol. 22, no. 5, pp. 598-604, 2006.

[15] T. A. Hillier, R. D. Abbott, and E. J. Barrett, "Hyponatremia: evaluating the correction factor for hyperglycemia," American Journal of Medicine, vol. 106, no. 4, pp. 399-403, 1999.

[16] R. Pethig and D. B. Kell, "The passive electrical properties of biological systems: their significance in physiology, biophysics and biotechnology," Physics in Medicine and Biology, vol. 32, no. 8, pp. 933-970, 1987.

[17] M. I. Lindinger, G. J. F. Heigenhauser, R. S. McKelvie, and N. L. Jones, "Blood ion regulation during repeated maximal exercise and recovery in humans," American Journal of Physiology, vol. 262, no. 1, pp. R126-R136, 1992.

[18] N. L. Anderson and N. G. Anderson, "The human plasma proteome: history, character, and diagnostic prospects," Molecular \& Cellular Proteomics, vol. 1, no. 11, pp. 845-867, 2002.

[19] J.-S. Park, J.-H. Choi, J.-J. Woo, and S.-H. Moon, "An electrical impedance spectroscopic (EIS) study on transport characteristics of ion-exchange membrane systems," Journal of Colloid and Interface Science, vol. 300, no. 2, pp. 655-662, 2006.

[20] M. I. Vázquez and J. Benavente, "A study of temperature effect on chemical, structural and transport parameters determined for two different regenerated cellulose membranes," Journal of Membrane Science, vol. 219, no. 1-2, pp. 59-67, 2003.

[21] M. Gourzi, A. Rouane, R. Guelaz et al., "Non-invasive glycaemia blood measurements by electromagnetic sensor: study in static and dynamic blood circulation," Journal of Medical Engineering and Technology, vol. 29, no. 1, pp. 22-26, 2005.

[22] A. Tura, S. Sbrignadello, D. Cianciavicchia, G. Pacini, and P. Ravazzani, "A low frequency electromagnetic sensor for indirect measurement of glucose concentration: in vitro experiments in different conductive solutions," Sensors, vol. 10, no. 6, pp. 5346-5358, 2010.

[23] S. Gabriel, R. W. Lau, and C. Gabriel, "The dielectric properties of biological tissues-II. Measurements in the frequency range $10 \mathrm{~Hz}$ to $20 \mathrm{GHz}$," Physics in Medicine and Biology, vol. 41, no. 11, pp. 2251-2269, 1996.

[24] P. Mirtaheri, S. Grimnes, and G. Martinsen, "Electrode polarization impedance in weak $\mathrm{NaCl}$ aqueous solutions," IEEE Transactions on Biomedical Engineering, vol. 52, no. 12, pp. 2093-2099, 2005.

[25] T. Ragheb and L. A. Geddes, "The polarization impedance of common electrode metals operated at low current density," Annals of Biomedical Engineering, vol. 19, no. 2, pp. 151-163, 1991.

[26] B. Rigaud, J.-P. Morucci, and N. Chauveau, "Bioelectrical impedance techniques in medicine-part I: bioimpedance measurement second section: impedance spectrometry," Critical Reviews in Biomedical Engineering, vol. 24, no. 4-6, pp. 257-351, 1997.

[27] C. Molteni and M. Parrinello, "Glucose in aqueous solution by first principles molecular dynamics," Journal of the American Chemical Society, vol. 120, no. 9, pp. 2168-2171, 1998.

[28] J. A. Te, M.-L. Tan, and T. Ichiye, "Solvation of glucose, trehalose, and sucrose by the soft-sticky dipole-quadrupoleoctupole water model," Chemical Physics Letters, vol. 491, no. 4-6, pp. 218-223, 2010.

[29] H. S. Kim, R. Pani, S. H. Ha, Y.-M. Koo, and Y. G. Yingling, "The role of hydrogen bonding in water-mediated glucose solubility in ionic liquids," Journal of Molecular Liquids, vol. 166, pp. 25-30, 2012. 

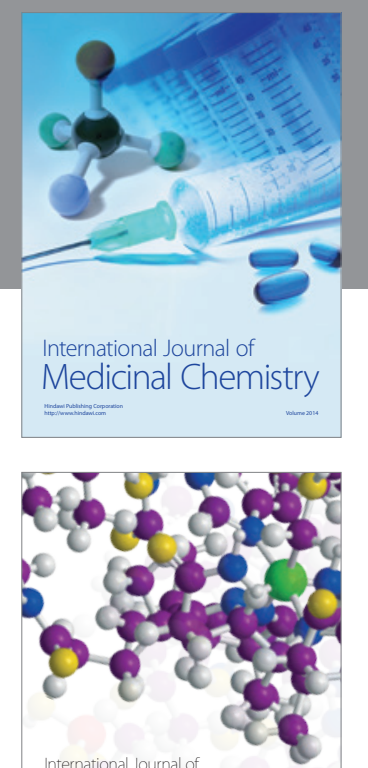

\section{Carbohydrate} Chemistry

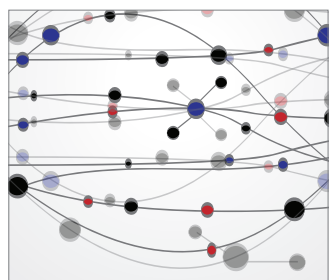

The Scientific World Journal
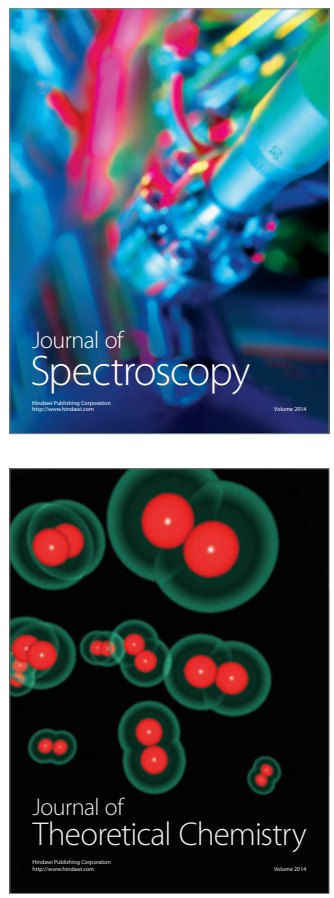
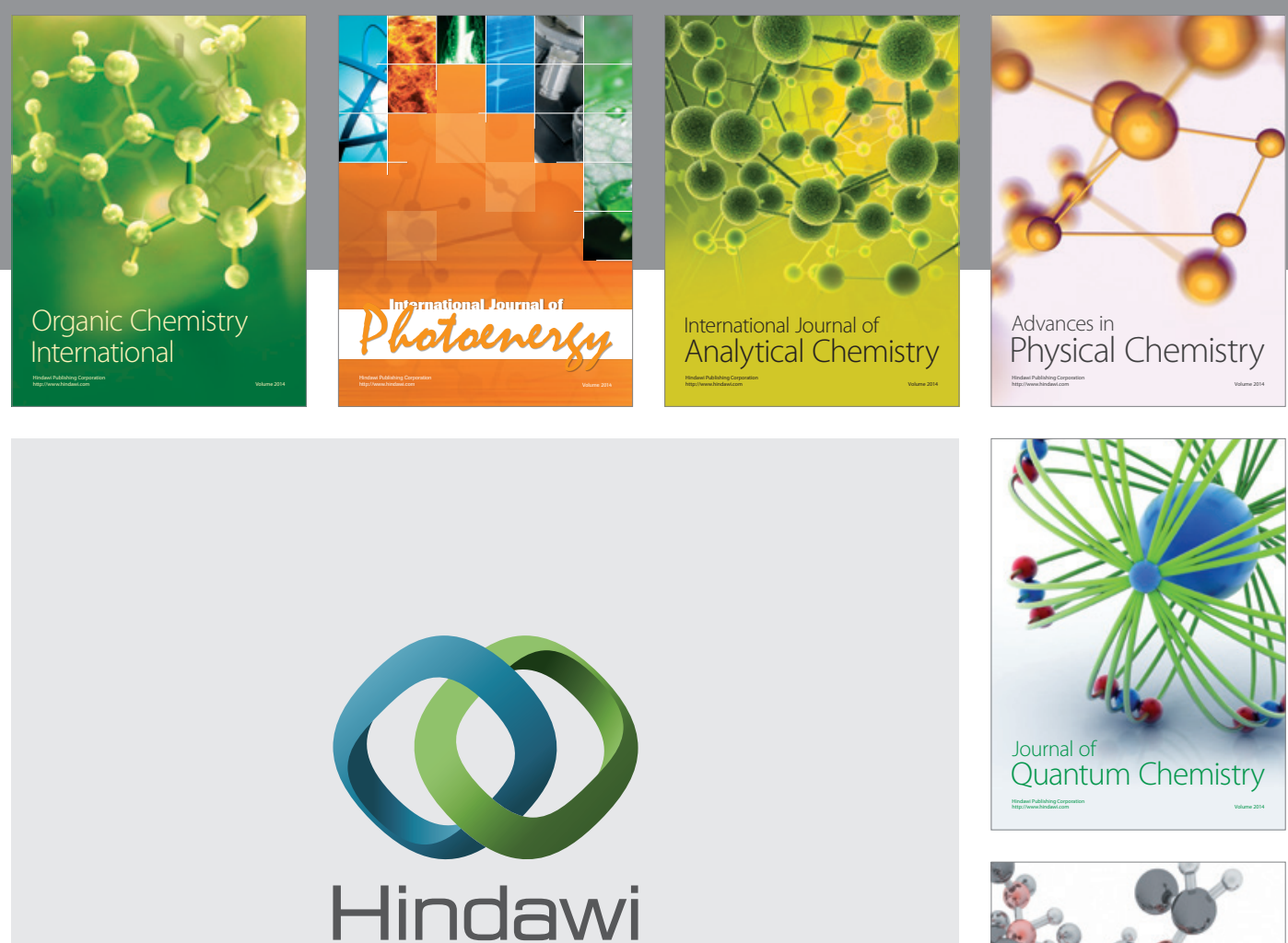

Submit your manuscripts at

http://www.hindawi.com

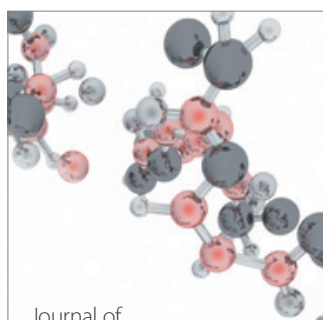

Analytical Methods

in Chemistry

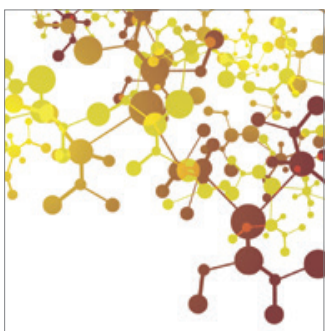

Journal of

Applied Chemistry

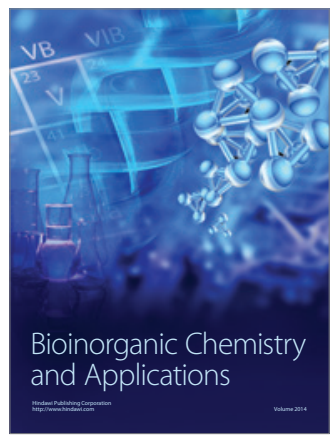

Inorganic Chemistry
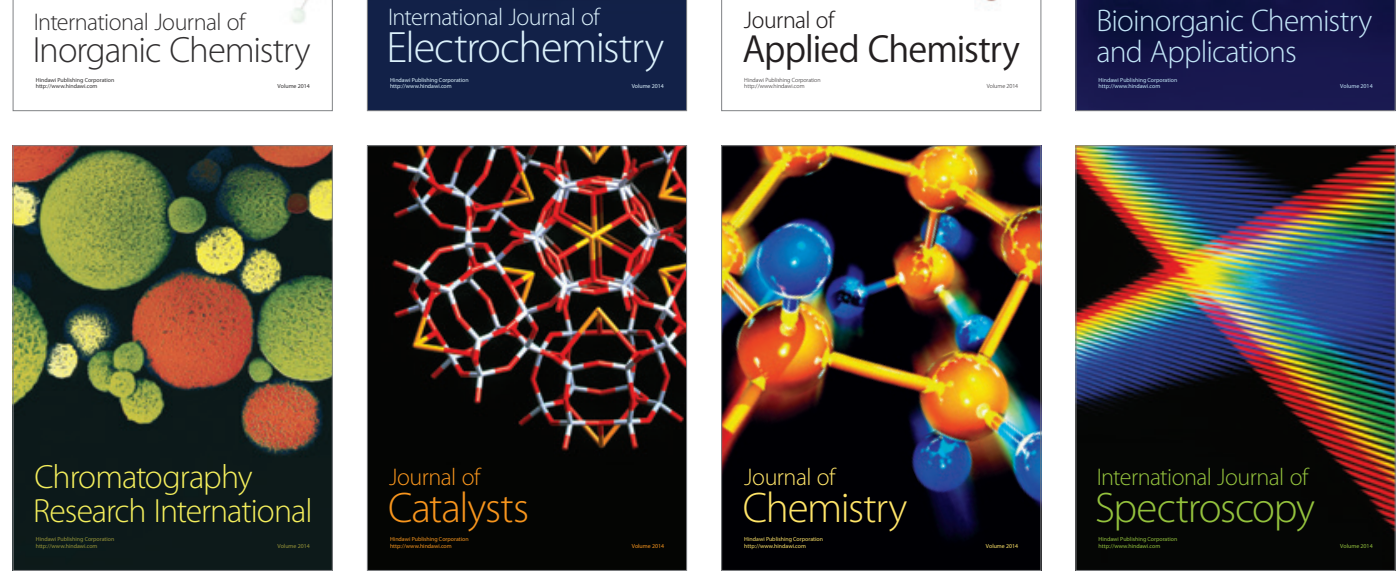